\title{
Drive-by-Wireless with the eCar Demonstrator
}

\author{
Hauke Stähle, Kai Huang, Alois Knoll
}

\begin{abstract}
Drive-by-wire technology has been gradually adopted by the car companies in recent years to integrate active assistance systems in vehicles to increase comfort and safety. To push the technologies for the electronic control systems of vehicles to extreme, we investigate the so-called drive-by-wireless, i.e., using wireless network to control steering, braking, accelerating, and other functions within an automobile. We use commercial off-the-shelf ZigBee MSP-EXP430F5438 Development Board for wireless communication and demonstrate our drive-by-wireless prototype on a 4-wheel steering/drive electric vehicle.
\end{abstract}

Keywords-Drive-by-Wireless, Electric Vehicles, Safety, Latency

\section{INTRODUCTION}

In recent years, drive-by-wire technology has been gradually adopted by the car companies to integrate active assistance systems in vehicles to increase comfort and safety [1]. Furthermore, complete new vehicle concepts are possible caused by the flexibility of driveby-wire technology. In general, those techniques replace the mechanical and hydraulic connections between the driver and the associated vehicle actuators with electronic communication systems. These systems transmit electronic messages to direct a vehicle component based on the action taken by the driver of the vehicle, e.g., turning a steering wheel, depressing a brake pedal, or depressing an accelerator pedal. The advantage of driveby-wire is that safety can be improved by providing computer controlled intervention of the driver's commands with systems such as crash avoidance, brake assistance, and lane assist systems.

To push further the technologies for the electronic control systems of vehicles, we investigate drive-bywireless, i.e., using wireless network, rather than electronic cables, to control steering, braking, accelerating, and other functions within an automobile. The advantage of using wireless is multifold:

- Eliminating wired connections between sensors, actuators, and control modules so as to, in turn, eliminate associated design, installation and maintenance costs of those wired connections [2]. For instance, the possibility of the malfunction of one or more wires may be eliminated through the use of a wireless network and the complexity of maintenance, problem solving, and repair may be reduced when wiring has been eliminated as a possible source of malfunction. In addition, the readiness of commercial off-the-shelf communications modules for wireless protocols, such as GSM, 3G, LTE, ZigBee, and WiFi, are available, which also

- Hauke Stähle, Kai Huang, Alois Knoll are with the Department of Informatics, Technische Universität München, Germany.

E-mail: firstname.lastname@in.tum.de results in simple design and lowered installation and maintenance costs.

- In some wired networks, all modules may share the same communication media. The capacity of the wired network may become congested and develop unacceptable latency. A wireless network for controlling vehicle functions may reduce the capacity limits associated with a wired network, e.g., using different frequency domains for different types of communication.

- A wireless network also may increase the flexibility of design options because sensor, actuators, and control modules may be located without regard for wiring requirements [3]. The installation of sensor modules and control modules may be easier when wires need not to be installed between a sensor module and a control module. This flexibility is important for designers and manufacturers, i.e., the simplification of assembly and the possibility of new modular design concepts.

- The integration of more and more safety functions increases the demand of inter-domain data transmission within the vehicle. The different domains of a car are connected nowadays via a central gateway which has to handle the cross-traffic of several bus systems with different technologies. Due to the availability of different channels for wireless communication, a node is not fixed to a specific domain anymore and can dynamically be grouped with senders and receivers. This eliminates the bottleneck of a central gateway within a vehicle.

Although drive-by-wireless conceptually provides lots of advantages, it also faces practical challenges. Two major problems are communication latency and safety. The latency issue comes from the additional delays which the wireless network induce due to the used protocol and propagation delay. The safety issue stems from the environment effects and interference from other communicating entities by which the wireless signal could be easily perturbed.

In this work, we mainly focus on the aforementioned 


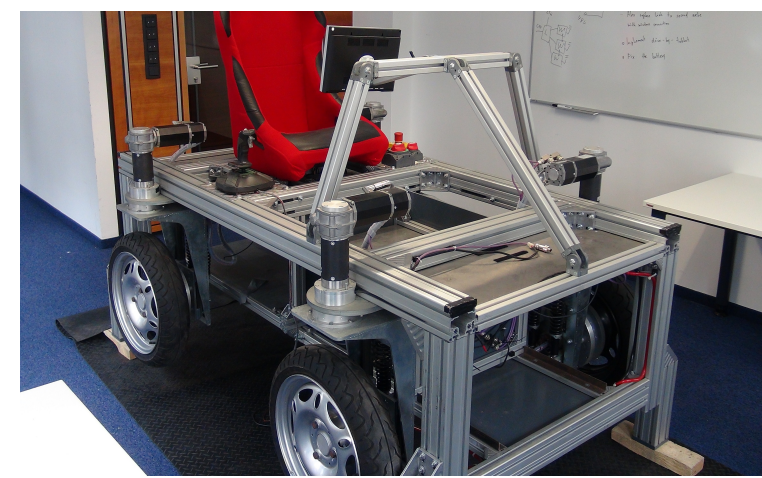

Fig. 1: fortiss eCar demonstrator - an experimental platform for innovative car architectures.

two challenges. A wireless deployment environment is setup based on a drive-by-wire four-wheel steering/drive electric vehicle (Fig. 1). To verify the concept of drive-by-wireless, ZigBee protocol is currently chosen to steer, accelerate, and decelerate one of the two axels. Commercial off-the-shelf MSP-EXP430F5438 Development Boards are used to integrate the ZigBee communication into the existing ICT infrastructure [4] of the eCar. To cope with interference between different invehicle ZigBee modules, a time division multiple access schedule is designed to provide temporal isolation. To tackle environmental effects and malicious hijacking, the beams of the eCar are used as a waveguide for the ZigBee communication. Our experimental results show that the communication latency and safety issues can be nicely tackled and the concept of drive-by-wireless is practical feasible on our vehicle.

The rest of this abstract is organized as follows: A short introduction for the fortiss eCar is presented in Section 2. Section 3 and Section 4 details our communication protocol and safety setup. Section 5 concludes the abstract.

\section{ECAR}

The complete drive power of the eCar [5] is $8 \mathrm{~kW}$. Each of the in-wheel engines has $2 \mathrm{~kW}$ and a maximum torque of $160 \mathrm{Nm}$. The eCar is controlled with a sidestick connected to the human-machine interface unit. The current state of the evaluation platform is presented on a 10-inch touchscreen, which can also be used as an input device, i.e., to change between different driving modes. The outline of the eCar is approximately $2.25 \times 1.25 \times 1.75$ $\mathrm{m}(\mathrm{L} \times \mathrm{W} \times \mathrm{H})$ and its weight is about $600 \mathrm{~kg}$. The eCar is constructed to carry one passenger.

The ICT infrastructure of the eCar, shown in Fig. 2, consists of four nodes: Three Texas Instrument LM3S8962 evaluation boards for the control functions of the eCar and one Intel Atom computer for the management of the human-machine interface. The evaluation boards are interconnected via Ethernet, running a real-time protocol. FreeRTOS is used as real-time operating system for the evaluation boards to manage the communication and

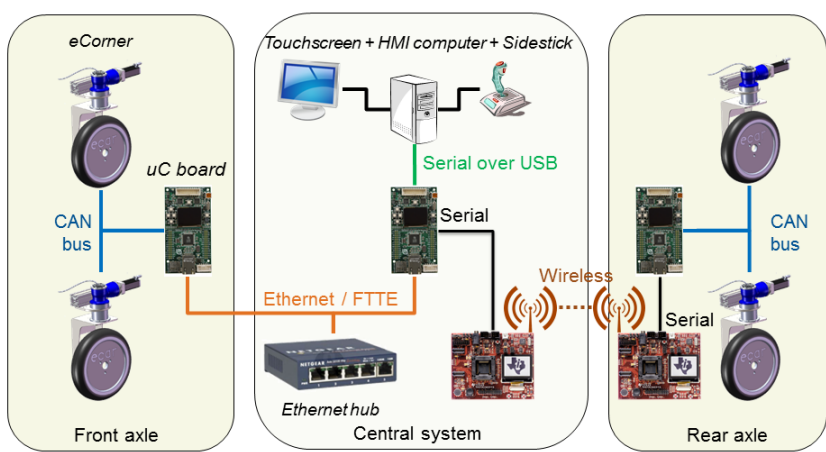

Fig. 2: Information and communication architecture of the eCar with wired and wireless data transmissions.

applications timings. The computer for the management of the human-machine interface runs Linux and shows an interface based on the Qt framework.

The connection between the evaluation boards and the motor and steering controllers is realized via CANBus (Controller Area Network), running the OpenCAN protocol. The central evaluation board and the humanmachine interface are connected via a serial link.

\section{Communication Latency}

The goal of our deployment setup is to replace all the Ethernet cables in the eCar with ZigBee wireless communication and achieving a comparable perfomance to the wired case.

In our first experiment, we replaced the Ethernet cable to the rear axle with a wireless connection while leaving the setup of the front axle untouched, see Fig. 2. This allows us to compare the perfomance of the two different approaches.

The wireless nodes are connected to the central controller and rear axle controller via a serial connection. The connection between the wireless host boards and the daughter boards with the actual transceiver is realized via Serial Peripheral Interface (SPI).

While the controllers of the front axle and the central system are synchronized via the flexible timetriggered ethernet (FTTE) protocol, the controllers of the central system and the rear axle are not, as the synchronization data is not forwarded via the wireless link. Nevertheless, the wireless system itself is running a beacon-based communication schedule to guarantee collision-free communication.

The schedule for the wireless communication is based on a beaconing approach. Although not necessary for the first experimental setup, the schedule was designed to serve a maximum of five nodes. The master node sends a beacon every $30 \mathrm{~ms}$ and the slave nodes respond after reception of the beacon and an individual delay, see also Fig. 2. The transmission time of one packet is $4 \mathrm{~ms}$ and after a silence phase of $2 \mathrm{~ms}$ for the processing ot the incoming data, the next node will send its data. Each packet has a size of 125bytes with 100bytes available 


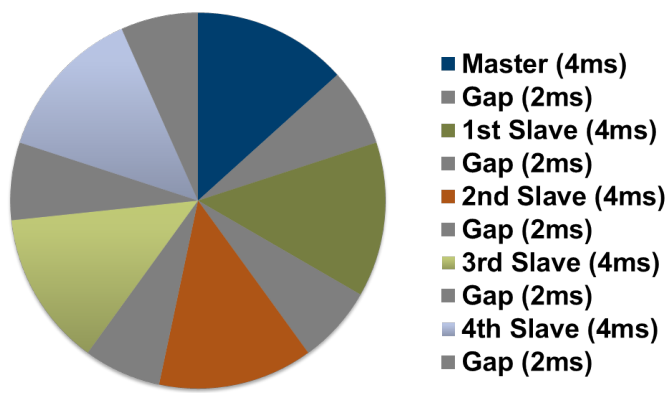

Fig. 3: The used time divison schedule for wireless data transmission.

for payload and is transmitted with $250 \mathrm{kbit} / \mathrm{s}$ over the wireless media. In case of a corrupted transmission, the packet is lost and not re-transmitted.

Fig. 4 shows the latency caused by other components of the system in the complete chain from the sidestick to the actuator. The communication between the hardware units is realized via buffers. Incoming data is stored in a buffer until a periodic task processes this data and writes the outgoing data into an output buffer. The data stays in the output buffer until a sending task picks it up and sends it via wire or wireless. The period of tasks processing incoming data is labeled "d_in" in Fig. 4 while the period of tasks transmitting the output buffers are labeled "d_out". A value of 0 indicates that the data is processed event-based. The latency of the controllers of the actuators is unknown, thus no values are given. Note that this representation is not sufficient to calculate a tight end-to-end delay as the interaction of different tasks within one controller are not considered.

First tests with this setup proved, that the achieved latency and transmission rate is suitable to control the rear axle of the eCar. In the near future this setup will be extended to also allow a wireless transmission to the front axle and the battery system making the ethernet based part obsolete. Also, we want to reduce the delay within the whole system to make it react faster to the driver's inputs.

As the slave nodes only react on the beaconing signal, a loss of the master node causes the complete wireless network to stop. Therefore, we want to extend our protocol to support redundancy via multiple transceivers and to be able to detect the absence of a beacon. In addition, we are developing a formal model for our setup, trying to provide a theoretical bound for the worst case end-to-end latency of our wireless communication.

\section{SAFETY}

The wireless transmission inside a vehicle is subject to electromagnetic interference. This can either be caused by other devices inside the vehicle, devices outside the vehicle or even caused by an external attack with the aim of a directed manipulation of the infrastracture.

The effect can reach from the degration of the communication perfomance up to a complete loss of the

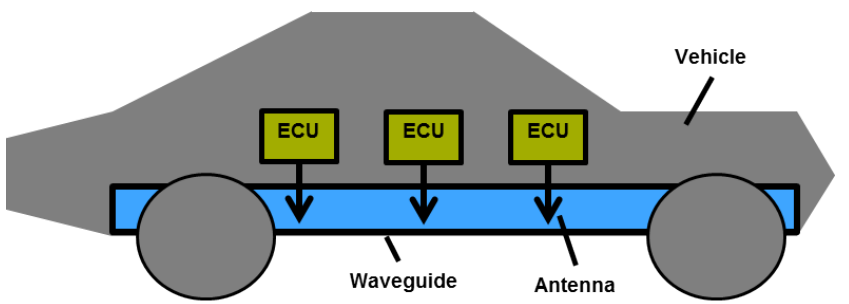

Fig. 5: Utilizing compartmens of the vehicle as waveguides.

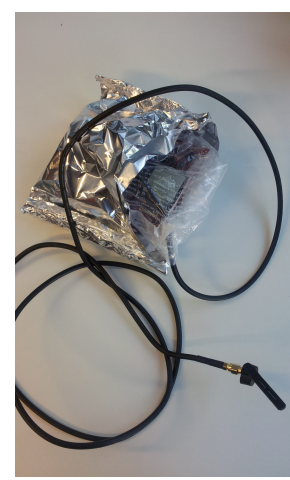

(a) Antenna extension

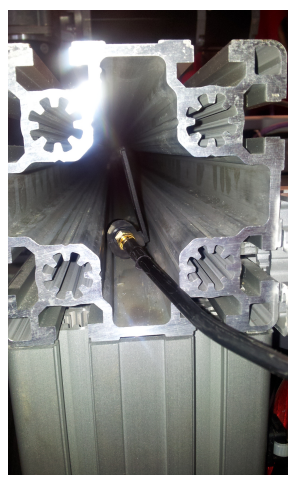

(b) Waveguide

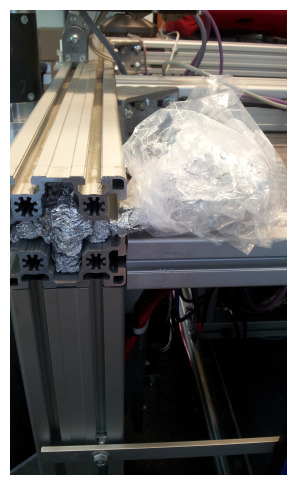

(c) Final setup
Fig. 6: Using the beam of the eCar as a waveguide. As there is an on-board antenna, a simplified insulation pack is built to shield signals from the on-board antenna.

communication ability. To prevent a malfunction of the system, we propose to use the internal compartments of the vehicle as a waveguide. This will hinder external signals to interfere with the wireless intra-vehicle communication. This concept is shown in Fig. 5.

With the availability of such a compartment that can act as a waveguide inside the vehicle, additional nodes can easily be integrated into the system by installing the antenna in the waveguide.

For our first experiments, we used the beams of the eCar as waveguides for the ZigBee communication to prevent malicious hijacking and to reduce environmental effects.

The experimental scenarios are shown in Tab. 1 . Basically, we record data acceptance rate between two ZibBee modules, under different combinations of: jammer on/off, antenna in/out of the beam, and end of the beam covered/open. The impacts of the distance and allocation of the jammer to the signal source are measured as well. In Fig. $7, j-\mathrm{C}-2$ represents that the jammer is two meters away from the signal source with the end of the beam covered with insulation materials (Here, $u$ represents an open end of the beam). j-c-10-a represents a $10 \mathrm{~cm}$ distance between the jammer and the signal source. The postfix $-a$ and $-b$ indicated different allocations between the jammer and source. As a signal jammer, we used another ZigBee node that sends random data continously on the same channel.

From Tab. 1, we can clearly see that the beam can 


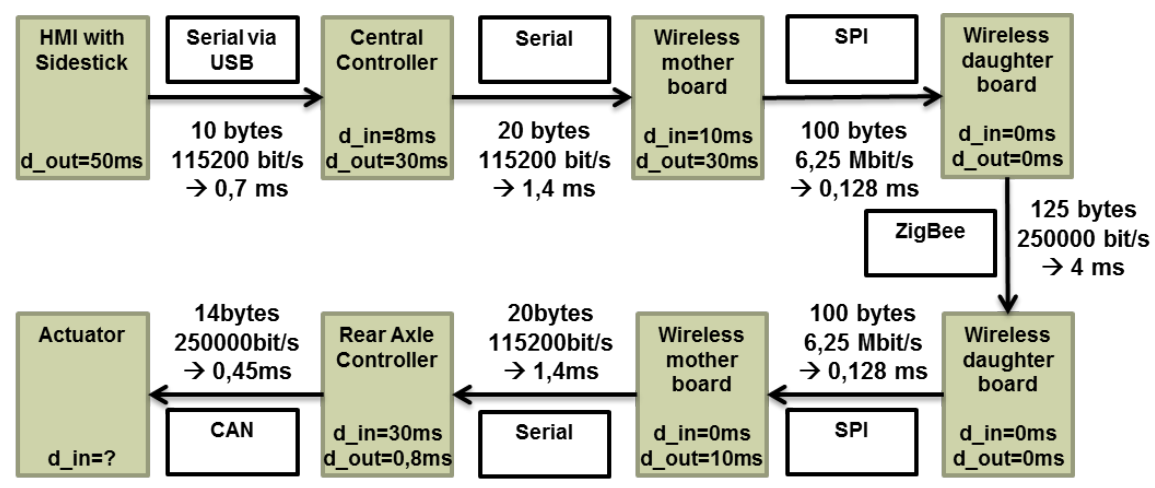

Fig. 4: Timings for the transmission of commands from the sidestick to the eCorner. d_in and d_out refer to the periodicity of the tasks processing the incoming and outgoing buffers, respectively.

TABLE 1: Experiment scenarios and results. The number indicate the acceptance rate. One antenna was always left inside the beam with an insulated beam end. The position of the other one was changed according to the table.

\begin{tabular}{|c|c|c|c|}
\hline \multicolumn{2}{|c|}{} & jammer off & jammer on \\
\hline antenna & covered & $100 \%$ & see Fig. 7 \\
\cline { 2 - 4 } inside beam & not covered & $100 \%$ & see Fig. 7 \\
\hline $\begin{array}{c}\text { antenna } \\
\text { outside beam }\end{array}$ & covered & $0 \%$ & $0 \%$ \\
\cline { 2 - 4 } & not covered & $100 \%$ & N/A \\
\hline
\end{tabular}

be used as an insulation media to protect the wireless signal from external interference for the scenario, where the open end of the beam is covered while the antenna of one device is outside the beam. The packet loss for this scenario is 100 percent.

The results shown in Fig. 7 are more interesting. In general, the jammer will affect the data acceptance rate. With the covering of the beam, the acceptance rate improved, which can be seen in the cases $j-c-2$, $j-c-10-a$, and $j-c-10-b$. However, the data acceptance rate cannot be recovered to $100 \%$ for all cases with a closed-end beam. The reason is, that there is an onboard antenna on the wireless module which cannot be put into the beam. Although we have made cover for the onboard antenna in order to insulate it, the signal is still affected. Another phenomenon is that the relative position between the jammer and signal source affects the acceptance rate as well, which is subject of further investigation.

\section{Conclusion}

We have proved with our experiments, that the concept of drive-by-wireless is feasible to control the eCar demonstrator. We tackled two of the main challenges towards a reliable drive-by-wireless concept: Latency issues by using a time-divison protocol for the wireless communication and safety issues by utilizing the beams of our demonstrator as waveguides to shield the wireless signals from external interference. The results show that the latency is low enough to control the eCar

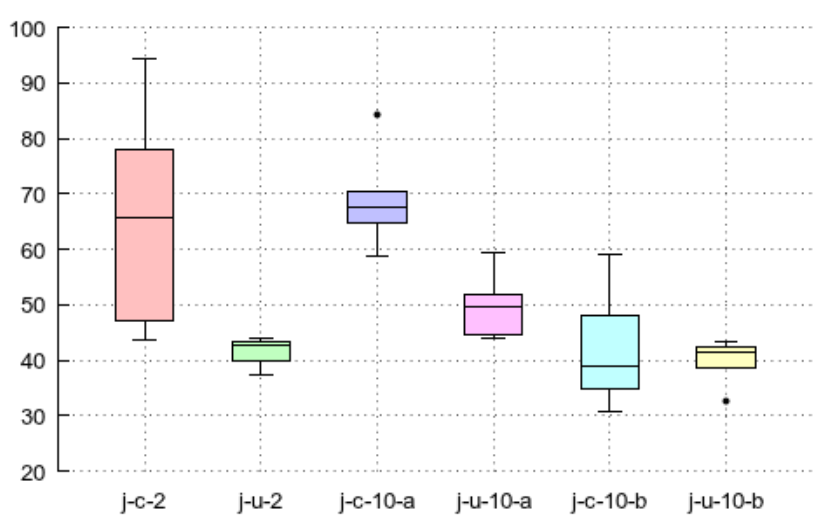

Fig. 7: Detailed results for the acceptance rates in different scenarios. $j=$ jammer active; $c=e$ nd of beam covered; $\mathrm{u}=\mathrm{end}$ of beam uncovered; $2=$ distance of $2 \mathrm{~m}$; $10=$ distance of $10 \mathrm{~cm} ; \mathrm{a}, \mathrm{b}=$ different positions of wireless nodes; the results are given as percentages.

and the concept of waveguides greatly improves the packet acceptance rates between the communication participants in the case of external interference.

In future work, we will investigate in an improvement of the shielding, a lowering of the latency to increase responsiveness and a comparison of suitable protocols for the in-vehicle use of wireless data transmission.

\section{REFERENCES}

[1] fortiss $\mathrm{GmbH}$, "The software car: Information and communication technology (ict) as an engine for the electromobility of the future," Tech. Rep., March 2011.

[2] M. Ahmed, C. Saraydar, T. ElBatt, J. Yin, T. Talty, and M. Ames, "Intra-vehicular wireless networks," in Globecom Workshops, 2007 IEEE, 2007, pp. 1-9.

[3] J.-R. Lin, T. Talty, and O. Tonguz, "Feasibility of safety applications based on intra-car wireless sensor networks: A case study," in Vehicular Technology Conference (VTC Fall), 2011 IEEE, 2011, pp. 1-5.

[4] C. Buckl, A. Camek, G. Kainz, C. Simon, L. Mercep, H. Staehle, and A. Knoll, "The software car: Building ict architectures for future electric vehicles," in Electric Vehicle Conference (IEVC), 2012 IEEE International, March 2012, pp. 1-8.

[5] M. Eder and A. Knoll, "Design of an experimental platform for an x-by-wire car with four-wheel steering," in Automation Science and Engineering (CASE), 2010 IEEE Conference on, 2010, pp. 656-661. 